\title{
Efeitos das Drogas Anti-Retrovirais sobre o Metabolismo Glicídico e Células de Langerhans de Pâncreas de Ratas Wistar Prenhes
}

\author{
Effects of Antiretroviral Drugs on Glucide Metabolism and Pancreatic Langerhans' Cells of \\ Pregnant Wistar Rats
}

Ernesto Antonio Figueiró-Filho, Patrícia El Beitune, Marilza Cunha Vieira Rudge, Silvana Maria Quintana, Alessandra Cristina Marcolin, Geraldo Duarte

\section{RESUMO}

Objetivo: avaliar a ação de drogas anti-retrovirais sobre o metabolismo glicídico e sobre o pâncreas de ratas Wistar prenhes.

Métodos: estudo com ratas prenhes adultas da raça Wistar, pesando entre 200-230 g. Foram testadas a azidotimidina, lamivudina e o nelfinavir, em doses 10 vezes superiores à dose utilizada em gestantes. Foram avaliados sete grupos, contendo 10 ratas por grupo, incluindo o controle. O sacrificio foi realizado no $21^{\circ}$ dia de prenhez. Procederam-se a dosagens de glicemia, insulina, glucagon, ácidos graxos livres (AGL) e glicogênio hepático. Para avaliação de lesão pancreática, optou-se pela contagem direta do número de células produtoras de insulina e glucagon marcadas por imuno-histoquimica. Os dados foram analisados pelo teste $t$ de Student, sendo comparados os animais dos grupos controle e tratados.

Resultados: após 21 dias de prenhez houve elevação dos niveis séricos de glucagon (grupo controle: $88,2 \mathrm{pg} / \mathrm{mL}$; grupos tratados: 99,7 a 120,7 pg/ mL) e redução dos niveis de insulina (grupo controle: 6,2 $\mu \mathrm{UI} / \mathrm{mL}$; grupos tratados: 2, 1 a 2,7 $\mu \mathrm{UI} / \mathrm{mL}$ ) em todos os grupos tratados com anti-retrovirais. Não houve diferenças significativas nos valores plasmáticos de glicemia, AGL e valores de glicogênio hepático ao final dos 21 dias de prenhez. Não houve diferença quanto ao número de células pancreáticas produtoras de insulina e glucagon entre os grupos tratados e o grupo controle ao final dos 21 dias de prenhez.

Conclusões: os fármacos anti-retrovirais utilizados durante a prenhez de ratas não infectadas alteram o metabolismo glicídico materno em grau leve causando queda de insulina e elevação do glucagon, com índices glicêmicos normais e número de células pancreáticas inalterado.

PALAVRAS-CHAVE: Drogas anti-retrovirais. Diabete melito. HIV. AIDS.

\section{Introdução}

O primeiro ensaio clínico no qual se administrou um agente anti-retroviral a gestantes para

Departamento de Ginecologia e Obstetrícia da Faculdade de Medicina de Ribeirão Preto da Universidade de São Paulo Correspondência:

Geraldo Duarte

Hospital das Clínicas da Faculdade de Medicina de Ribeirão Preto da Universidade de São Paulo

Avenida Bandeirantes, 3900

14049-900 - Ribeirão Preto - SP

Fone: (16) 602-2588

e-mail: gduarte@.fmrp.usp.br avaliação de sua segurança, tolerância e eficácia na redução da transmissão vertical (TV) do vírus da imunodeficiência tipo 1 (HIV-1) concluiu que a utilização de azidotimidina (AZT) durante o período gestacional e durante o trabalho de parto, além do uso dessa medicação pelo recém-nascido, pôde reduzir a TV do virus em $67,5 \%{ }^{1}$.

Os avanços obtidos a partir do entendimento da patogênese da transmissão perinatal do HIV1 e sua redução com o uso do AZT estimularam o estudo de novas drogas com potente ação antiretroviral que pudessem ser utilizadas com segurança no período gestacional ${ }^{2}$. São escassas, no 
entanto, as informações sobre doses, farmacocinética e efeitos colaterais dessas drogas durante a gestação, mesmo na atualidade ${ }^{3,4}$.

Fica evidente a preocupação de inúmeros autores quanto ao efeito dos medicamentos antiretrovirais sobre o metabolismo de carboidratos e lipídios, sendo os inibidores de protease os principais protagonistas destes efeitos deletérios sobre o organismo humano ${ }^{5,6}$. Entretanto, pesquisas destes efeitos durante a gestação ainda são pouco freqüentes, sendo evidente a necessidade de estudos enfatizando estas alterações em gestantes portadoras do HIV-1 ${ }^{7,8}$.

Modelos de estudos experimentais sobre a infecção pelo HIV-1 utilizam animais infectados por oncovirus indutores de leucemia ou mutados geneticamente, com a finalidade de avaliar a eficácia, efeitos terapêuticos e colaterais das drogas anti-retrovirais ${ }^{9}$. Experimentalmente, até o momento atual, não foram encontrados trabalhos nos quais se avalia o efeito dos fármacos antiretrovirais usualmente utilizados em gestantes sobre o metabolismo glicídico de animais expostos durante a prenhez. São disponíveis, entretanto, trabalhos experimentais diversos com ratas prenhes, avaliando o efeito de outras drogas com potencial sabidamente diabetogênico ${ }^{10-12}$.

Nos animais que desenvolvem diabete melito experimentalmente observa-se aumento significativo dos níveis glicêmicos ${ }^{10-12}$ e elevação de ácidos graxos livres ${ }^{12,13}$, fato significativo da ação diabetogênica dos fármacos utilizados. A dosagem de glicogênio em fragmento de figado após sacrificio de animais diabéticos demonstra importante redução ${ }^{12,14}$. Observam-se também redução dos niveis de insulina e elevação do glucagon nos casos de lesão pancreática e desenvolvimento de diabetes experimental ${ }^{11,15,16}$.

Adicionalmente, pode-se avaliar o efeito diabetogênico de medicamentos diretamente sobre o tecido pancreático utilizando a observação histológica das ilhotas de Langerhans marcadas com uso de imuno-histoquímica. Ocorrendo alterações de dosagens laboratoriais, pode-se confirmar e quantificar a extensão do dano pancreático pela avaliação das células produtoras de insulina e glucagon. A técnica de imuno-histoquímica é o método de escolha utilizado por muitos pesquisadores para confirmação de danos diretos sobre o pâncreas, nos estudos experimentais para avaliação de efeitos diabetogênicos de substâncias diversas. Usualmente são utilizados anticorpos primários antiglucagon $(1: 10.000)$ e antiinsulina $(1: 1000)$ e anticorpos biotinilados anticoelho (1:200), para glucagon, e anticobaia (1:300), para insulina ${ }^{11.15,16}$.
Após revisão bibliográfica sobre os principais agentes anti-retrovirais e seus efeitos sobre o organismo materno e fetal, observou-se a importância de estudos experimentais nos quais pode-se testar os efeitos farmacológicos e adversos das drogas anti-retrovirais em modelos animais. Verificou-se que lacunas existem no conhecimento dos efeitos das drogas antiretrovirais sobre o metabolismo glicídico durante a gestação e, até o presente, modelos experimentais para avaliar a ação diabetogênica dessas drogas em ratas prenhes não infectadas ainda não foram testados.

Desse modo, objetivou-se com o presente estudo a avaliação do efeito de drogas antiretrovirais administradas isoladamente e em associação sobre o pâncreas de ratas prenhes expostas a estes fármacos, utilizando dosagens laboratoriais e avaliação imuno-histoquímica do tecido pancreático.

\section{Métodos}

Estudo experimental realizado no Biotério do Departamento de Clínica Médica da Faculdade de Medicina de Ribeirão Preto da Universidade de São Paulo (FMRP-USP). Foram utilizadas ratas fêmeas prenhes da raça Wistar, com peso inicial entre 200 e 230 g. Os animais foram divididos aleatoriamente em sete grupos com 10 animais em cada, sendo um controle e seis grupos que receberam medicamentos anti-retrovirais isolados ou em associação. Optou-se por trabalhar com as drogas anti-retrovirais mais utilizadas durante a gestação: AZT, lamivudina (3TC) e nelfinavir (NFV). Tais fármacos foram fornecidos e fracionados pela farmácia do Hospital das Clínicas da FMRP-USP. As suas dosagens foram padronizadas em 10 vezes a dose utilizada em pacientes gestantes, proporcionalmente ao peso dos animais. Desse modo as ratas receberam doses de $25 \mathrm{mg} /$ dia de AZT, 12,5 $\mathrm{mg} /$ dia de 3TC e $97,5 \mathrm{mg} /$ dia de NFV.

A via de administração dos anti-retrovirais foi oro-gástrica, utilizando a técnica de gavagem, após diluição dos medicamentos em $5 \mathrm{~mL}$ de solução fisiológica a 0,9\%. O grupo controle recebeu solução fisiológica na mesma proporção. A administração foi sempre pela manhã, em dose única diária.

Uma vez que o período de prenhez de ratos tem duração de 21 dias, a análise dos efeitos das drogas sobre o organismo dos animais e de suas crias foi realizada no $21^{\circ}$ dia de prenhez. A experimentação teve início no "dia zero" da prenhez de 
todos os animais de cada grupo. A prenhez foi diagnosticada pela análise microscópica do esfregaço vaginal, sendo a observação da presença de espermatozóides no esfregaço o indicador do "dia zero" da prenhez. Os animais receberam água e alimentação ad libitum antes e durante o período de prenhez.

No $21^{\circ}$ dia de prenhez os animais foram sacrificados por decapitação e o sangue proveniente da área cruenta cervical, imediatamente colhido. Essa amostra sangüínea foi processada para realização de dosagens plasmáticas de glicemia e ácidos graxos livres (AGL) e dosagens séricas de lactato, insulina e glucagon. Todo o processo de coleta sanguínea, separação de soro e plasma foi realizado sob refrigeração constante.

Após o sacrifício de cada animal e tendo sido coletado o sangue, procedeu-se à laparotomia, em prazo inferior a 5 minutos pós-morte, objetivando a retirada de fragmento de figado para dosagem de glicogênio hepático. Foram retirados, ainda, dois fragmentos pancreáticos, sendo um junto ao baço (cauda pancreática) e outro junto ao duodeno (cabeça pancreática, região superior), imediatamente fixados em formol tamponado. Este material foi incluído em parafina para realização da imunohistoquímica, com localização de células produtoras de glucagon (células alfa) e insulina (células beta) pela técnica do complexo avidina-biotinaperoxidase (ABC - Vector Laboratories, USA), com modificações ${ }^{17}$. Em todos os tecidos pancreáticos aplicou-se o método de recuperação de epítopos utilizando o forno de microondas (Electrolux, 900W) em solução de ácido cítrico, pH 6,0, por 15 min. Os tecidos fixados em formalina e incluídos em parafina foram cortados com $3 \mu \mathrm{m}$ de espessura e montados em lâminas de vidro previamente preparadas com o adesivo poli-D-lisina (Sigma Chemical Corporation, USA) para evitar o descolamento dos cortes durante a imunocoloração. Em seguida, os cortes foram desparafinizados em xilol por $5 \mathrm{mi}-$ nutos (3 banhos), hidratados em banhos com álcool de concentração decrescente (4 banhos) e lavados em solução salina tamponada (SST) em pH 7,4 durante 5 minutos. Posteriormente, os cortes foram tratados com peróxido de hidrogênio $\left(\mathrm{H}_{2} \mathrm{O}_{2}\right)$ a $3 \%$, diluído em SST por 5 minutos, para bloqueio da peroxidase endógena. Após a recuperação de epítopos, os tecidos foram incubados com anticorpos primários antiglucagon $(1: 10.000)$ (Diasorin, Inc., USA) e antiinsulina $(1: 1000)$ (Dakocytomation, USA), durante um periodo médio de 16 horas incluindo o período noturno em temperatura de aproximadamente $4^{\circ} \mathrm{C}$ em refrigerador. No dia seguinte, após lavagem em SST, os cortes foram incubados com anticorpo secun- dário biotinilado anticoelho (1:200) (Vector Laboratories), para glucagon, e anti-cobaia (1:300) (Vector Laboratories), para insulina, por $60 \mathrm{minu}-$ tos. A seguir, realizou-se incubação das lâminas com os reagentes do sistema de detecção $\mathrm{ABC}$, conforme especificação do fabricante. Para leitura da reação, todos os cortes foram tratados com solução de DAB [(3-3)-tetraidrocloreto de diaminabenzidina] na concentração de $1 \mathrm{mg} / \mathrm{mL}$ de solução tampão de Tris e solução de $\mathrm{H}_{2} \mathrm{O}_{2}$ por 5 minutos. A seguir os cortes foram contracorados com hematoxilina de Harris por 5 minutos, com posteriores banhos de concentrações crescentes de álcool etílico ( 5 banhos) e xilol (3 banhos). Todos os passos da reação imuno-histoquímica foram realizados à temperatura ambiente, com exceção da incubação com os anticorpos primários (temperatura aproximada de $4^{\circ} \mathrm{C}$ ) e para recuperação de epítopos. Entre cada passo da reação as lâminas foram lavadas várias vezes com SST $(\mathrm{pH}=7,4)$.

Após coradas e identificadas, as lâminas foram observadas em microscópio óptico comum, tendo sido localizadas as dez melhores ilhotas de Langerhans em cada lâmina, de acordo com a coloração e integridade das células. As células beta localizam-se no centro das ilhotas, ao passo que as células alfa são localizadas na sua periferia ${ }^{18}$. As células marcadas com os anticorpos citados adquirem coloração acastanhada e são facilmente contadas e identificadas. Para avaliação objetiva de dano tecidual, optou-se pela contagem direta do número de células produtoras de insulina e glucagon de cada animal em estudo, utilizando objetiva com aumento máximo sem imersão (40X). Depois de selecionadas as dez melhores ilhotas, as células foram contadas, uma a uma, sendo cada ilhota focada individualmente no campo óptico. A contagem das células foi realizada com o auxílio do software Image Tool for Windows da UTHSCSA (University of Texas Health Science Center in San Antonio, versão 3.00, Copyright 19952002). Desse modo, de cada animal obteve-se um número médio de células produtoras de insulina e glucagon a partir da contagem de células de dez ilhotas por animal. As células foram contadas tanto nos animais do grupo controle quanto naqueles submetidos à administração de drogas anti-retrovirais.

Os dados obtidos de dosagens laboratoriais, o peso materno e a média obtida de células pancreáticas foram submetidos à análise estatística por meio do teste $t$ de Student para comparação de dois grupos (controle $v$ s tratados). Foram considerados valores estatisticamente significativos quando o valor de $\mathrm{p}$ bicaudal foi menor que 0,05 . 
Resultados

Objetivando sintetizar as alterações mais importantes, a Tabela 1 demonstra que após 21 dias de prenhez o grupo controle apresentou valor médio de $88,2 \mathrm{pg} / \mathrm{mL}$ para glucagon e $6,2 \mu \mathrm{UI} / \mathrm{mL}$ para insulina, os grupos que receberam drogas anti-retrovirais apresentaram variações compreendidas entre 2,1 a $2,7 \mu \mathrm{UI} / \mathrm{mL}$ para insulina e 99,7 a 120,7 pg/mL para glucagon. Esses valores demonstram haver elevação dos níveis séricos de glucagon e redução dos níveis de insulina em todos os grupos tratados com anti-retrovirais em relação ao grupo controle (teste $t$ de Student, $\mathrm{p}<$ $0,05)$.

Tabela 1 - Valores séricos de insulina e de glucagon no $21^{\circ}$ dia de prenhez.

\begin{tabular}{lcc}
\hline Grupo & Insulina $(\mu \mathrm{Ul} / \mathrm{mL})$ & Glucagon $(\mathrm{pg} / \mathrm{mL})$ \\
\hline Controle & $6,2 \pm 0,9$ & $88,2 \pm 5,5$ \\
AZT & $2,7 \pm 1,4^{*}$ & $109,2 \pm 22,7^{*}$ \\
$3 T C$ & $2,3 \pm 1,8^{*}$ & $113,6 \pm 36,0^{*}$ \\
NFV & $2,2 \pm 1,5^{*}$ & $114,4 \pm 31,6^{*}$ \\
AZT + 3TC & $2,1 \pm 1,5^{*}$ & $116,2 \pm 21,9^{*}$ \\
AZT + NFV & $2,1 \pm 1,4^{*}$ & $99,7 \pm 10,5^{*}$ \\
AZT + 3TC + NFV & $2,1 \pm 1,1^{*}$ & $120,7 \pm 37,3^{*}$ \\
\hline
\end{tabular}

${ }^{*} p<0,05$. Controle $x$ Medicamentos.

Teste $t$ de Student.

Valores expressos em média $\pm \mathrm{DP}$

Grupos com $n=10$ animais.

Não houve diferenças significativas nos valores plasmáticos de glicemia (controle: $87,5 \mathrm{mg}$ / $\mathrm{dL}$; tratados: 83,7 a $95,5 \mathrm{mg} / \mathrm{dL}$ ) e AGL (controle: $0,43 \mu \mathrm{mol} / \mathrm{mL}$; tratados: 0,31 a $0,44 \mu \mathrm{mol} / \mathrm{mL}$ ), bem como nos valores de glicogênio hepático ao final dos 21 dias de prenhez. (Tabela 2).

Tabela 2 - Valores plasmáticos de glicemia e AGL e de glicogênio hepático no $21^{\circ}$ dias de prenhez.

\begin{tabular}{llcc}
\hline Grupo & $\begin{array}{c}\text { Glicemia } \\
(\mathrm{mg} / \mathrm{dL})\end{array}$ & $\begin{array}{c}\text { AGL } \\
(\mu \mathrm{mol} / \mathrm{mL})\end{array}$ & $\begin{array}{c}\text { Glicogênio } \\
\text { hepático }(\%)\end{array}$ \\
\hline Controle & $87,5 \pm 12,5$ & $0,4 \pm 0,1$ & $3,1 \pm 1,3$ \\
AZT & $95,5 \pm 7,3$ & $0,4 \pm 0,1$ & $3,8 \pm 0,8$ \\
3TC & $86,8 \pm 13,4$ & $0,4 \pm 0,1$ & $3,4 \pm 1,0$ \\
NFV & $83,7 \pm 15,2$ & $0,3 \pm 0,1$ & $3,7 \pm 1,0$ \\
AZT + 3TC & $84,0 \pm 20,1$ & $0,4 \pm 0,1$ & $3,9 \pm 0,8$ \\
AZT + NFV & $87,0 \pm 8,4$ & $0,4 \pm 0,1$ & $3,4 \pm 0,3$ \\
AZT + 3TC + NFV & $86,5 \pm 11,3$ & $0,3 \pm 0,1$ & $3,9 \pm 0,5$ \\
\hline
\end{tabular}

$p>0,05$. Controle vs Medicamentos.

Teste $t$ de Student.

Valores expressos em média \pm DP.

Grupos com $n=10$ animais.

AGL - ácidos graxos livres.
Embora houvesse redução significativa dos valores séricos de insulina e elevação dos valores de glucagon nos grupos que receberam medicação anti-retroviral em relação ao grupo controle (Tabela 1), não houve diferenças quanto ao número de células pancreáticas produtoras de insulina (controle: 160 células/animal; tratados: 162 a 170 células / animal) ao final dos 21 dias de prenhez. O número de células pancreáticas produtoras de glucagon também não apresentou diferenças significativas (controle: 115,6 células / animal; tratados: 112,5 a 116,3 células /animal) (Tabela 3).

Tabela 3 - Número de células produtoras de insulina e glucagon em pâncreas de ratas após 21 dias de prenhez.

\begin{tabular}{lcc}
\hline Grupo & \multicolumn{2}{c}{ Número de células } \\
Insulina & Glucagon \\
\hline Controle & $160,0 \pm 48,8$ & $115,6 \pm 25,8$ \\
AZT & $170,1 \pm 59,3$ & $113,7 \pm 20,8$ \\
$3 T C$ & $170,0 \pm 53,5$ & $112,2 \pm 21,6$ \\
NFV & $159,1 \pm 54,1$ & $116,3 \pm 21,1$ \\
AZT + 3TC & $170,0 \pm 50,8$ & $114,9 \pm 24,3$ \\
AZT + NFV & $162,3 \pm 54,8$ & $112,5 \pm 21,4$ \\
AZT + 3TC + NFV & $169,4 \pm 53,3$ & $115,8 \pm 20,9$ \\
\hline
\end{tabular}

$p>0,05$. Comparações: Controle vs Medicamentos.

Teste $t$ de Student.

Valores expressos em média $\pm \mathrm{DP}$

Células contadas em 10 ilhotas de Langerhans/animal.

Grupos com $n=10$ animais.

\section{Discussão}

Em trabalhos experimentais, observa-se aumento significativo dos niveis glicêmicos nos animais que desenvolvem diabete melito experimentalmente com a utilização de medicamentos indutores de lesão pancreática ${ }^{10-12}$. No presente estudo não foram observadas alterações significativas nos niveis de glicemia dos animais que receberam medicamentos anti-retrovirais em relação aos do grupo controle após 21 dias de prenhez.

Entretanto, dentre os parâmetros analisados, observou-se que os valores de insulina sofreram redução e os níveis séricos de glucagon sofreram elevação em todos os grupos de animais tratados em relação ao controle, ao final dos 21 dias de prenhez. As dosagens hormonais de insulinemia e glucagonemia são auxiliares no acompanhamento do efeito diabetogênico nos animais estudados, ocorrendo redução dos níveis insulinêmicos e elevação dos níveis do glucagon nos casos de lesão pancreática e desenvolvimento de diabete melito tipo $1^{11,15,16}$. 
Apesar dos valores normais de glicemia verificados nos animais do presente estudo, as alterações nos níveis séricos de insulina e glucagon indicam efeito sobre o pâncreas materno. A redução da insulina sérica como marcador de lesão pancreática é relatada em todos os estudos com ratos diabéticos, nos quais houve comprovação de lesão de ilhotas de Langerhans por meio de imunohistoquímica pancreática com anticorpos anti-insulina $^{11,15,16}$, e revela redução significativa do número de células produtoras de insulina e aumento de células produtoras de glucagon. Tal observação confirma a relação existente entre dosagens hormonais alteradas e efeito medicamentoso lesivo sobre o pâncreas.

Em recente estudo, Kanter et al. ${ }^{11}$ demonstraram a relação direta entre hiperglicemia e redução sérica de insulina materna com lesão pancreática e redução no número de células produtoras de insulina em ratas prenhes com diabete induzido com estreptozotocina. Nos fetos, os mesmos autores demonstraram hiperinsulinemia e aumento do número de células beta nas ilhotas de Langerhans utilizando avaliação imuno-histoquímica pancreática materna e fetal. Observa-se, portanto uma relação entre valores séricos circulantes de insulina e número de células pancreáticas.

Do mesmo modo, em estudo no qual se avaliaram hamsters com diabete induzido por estreptozotocina, demonstrou-se aumento de células alfa produtoras de glucagon nos pâncreas dos animais. Os autores indicam a relação direta entre o aumento celular pancreático e a hiperglucagonemia encontrada ${ }^{19}$.

Também avaliando o número de células pancreáticas produtoras de insulina e glucagon em animais ao longo da prenhez e no periodo puerperal, descreveram-se diminuição de células beta e aumento de células alfa em ratas com diabete induzido por estreptozotocina. Esses animais demonstraram queda de insulina e elevação de glucagon séricos. Entretanto, os mesmos autores também demonstraram que alguns animais, apesar dos níveis séricos alterados de insulina e glucagon, demonstraram números de células alfa e beta inalterados em relação ao grupo controle ${ }^{20}$.

Apesar de os niveis glicêmicos dos animais da presente casuística serem normais, a redução da insulina e elevação do glucagon pode ter significado efeito diabetogênico ainda inicial, uma vez que não houve danos celulares pancreáticos nas células produtoras de insulina ou glucagon, após 21 dias de prenhez, nos animais tratados com drogas antiretrovirais, em relação ao grupo controle.

Alterações semelhantes aos resultados encontrados no presente estudo também já foram demonstradas por outros autores, com relatos de que, após 14 dias da aplicação de doses mais baixas de estreptozotocina em ratos, os animais apresentaram-se normoglicêmicos e com número de células beta inalterados, apesar da redução dos níveis pancreáticos e séricos de insulina ${ }^{21}$.

Em animais nos quais houve administração de estreptozotocina com dois dias de vida, não houve aparecimento de hiperglicemia com dez dias de vida, apesar dos níveis séricos reduzidos de insulina. As ilhotas pancreáticas apresentaram-se normais quanto ao número de células produtoras de insulina e glucagon, sugerindo recuperação parcial pancreática quando ocorre indução diabetogênica precoce. Desse modo, apesar de o número de células estar inalterado, a dosagem de insulina pancreática demonstrou-se reduzida ${ }^{22}$.

Demonstrou-se que ocorre regeneração de células beta pancreáticas em animais com diabete induzido com altas doses de estreptozotocina. Tal regeneração celular é ainda maior quando os animais são tratados com insulina exógena, chegando o número de células produtoras de insulina a alcançar $40 \%$ dos valores descritos no grupo controle. Esses resultados revelam que mesmo os pâncreas farmacologicamente lesados são passíveis de regeneração celular, a qual aumenta se o controle glicêmico for adequado ${ }^{23}$.

Ratas normais apresentam aumento progressivo de tecido pancreático ao longo da prenhez, com elevação do número de células beta, aumento de secreção insulínica pancreática, elevação dos níveis séricos de insulina e conseqüentemente niveis glicêmicos progressivamente mais baixos conforme evolui a prenhez. No período de lactação observam-se queda nos níveis séricos de insulina, diminuição do número de células pancreáticas e manutenção da glicemia. Esses resultados experimentais demonstram claramente que a prenhez estimula a gênese de células pancreáticas, objetivando o aumento de secreção insulínica e manutenção da glicemia ${ }^{24}$.

Os medicamentos anti-retrovirais, quando administrados de forma associada (AZT + 3TC + NFV) a pacientes gestantes infectadas pelo vírus HIV-1, ocasionam intolerância glicêmica, no entanto, não causam efeitos deletérios sobre o prognóstico perinatal ${ }^{7}$. A administração de AZT isoladamente, não associada a outros anti-retrovirais, não causa alterações no metabolismo glicídico ${ }^{7}$.

Os resultados aqui expostos demonstram que as drogas anti-retrovirais causam alterações no metabolismo de carboidratos de ratas prenhes submetidas aos efeitos dessas drogas durante o periodo de prenhez. Apesar de os animais não terem desenvolvido hiperglicemia e de não ter ha- 
vido lesão pancreática tecidual, foram observadas alterações importantes do metabolismo glicídico, comprovadas pela redução dos níveis de insulina e elevação dos valores de glucagon nos animais tratados em relação ao grupo controle.

Pode-se afirmar que houve efeito diabetogênico induzido pelos fármacos antiretrovirais nos animais prenhes avaliados, mesmo com índices glicêmicos normais e número de células pancreáticas inalterado. A hiperglicemia é o último parâmetro a ser observado em animais com diabete induzido, sendo precedida por redução dos níveis de insulina pancreática e sérica ${ }^{25}$. O número inalterado de células pancreáticas encontrado nos animais do presente estudo pode ser justificado pelas adaptações orgânicas impostas pela prenhez em ratos, associando-se ainda à capacidade regenerativa pancreática desses animais diante de situações de lesão tecidual ${ }^{23,24}$.

Ratas submetidas a única dose de estreptozotocina 14 dias antes da prenhez demonstraram queda de insulina pancreática e redução do número de células beta. Todavia, após prenhes, houve recuperação de $60 \%$ do tecido pancreático lesado e normalização dos níveis pancreáticos de insulina, comprovando que a prenhez induz a recuperação pancreática nesses animais ${ }^{26}$.

Os resultados aqui expostos demonstram que os fármacos anti-retrovirais, quando utilizados durante a prenhez de ratas não infectadas, alteram o metabolismo glicídico, ainda que em graus iniciais, abrindo várias possibilidades de estudos futuros. Alguns questionamentos podem ser levantados a respeito desses resultados: a utilização dos medicamentos anti-retrovirais em ratas, somente a partir do diagnóstico de prenhez, poderia ter reduzido o efeito diabetogênico desses fármacos devido às adaptações metabólicas que ocorrem nesse período? Um tempo de exposição maior aos fármacos anti-retrovirais, não apenas durante o período de prenhez, poderia causar danos maiores e conseqüente diminuição de células beta, além da alteração de outros parâmetros metabólicos nos animais estudados?

Evidências clínicas e experimentais demonstram que a homeostase materna é a principal responsável pelo controle do diabete durante a gravidez, sendo que o adequado equilíbrio metabólico materno garante a redução da morbimortalidade fetal $^{27}$. Justificam-se, então, pesquisas que busquem fatores de risco para o desenvolvimento de hiperglicemia no período gestacional, de forma a se fazer diagnóstico precoce ou instituir medidas profiláticas durante o pré-natal que possam garantir o bem estar do feto, melhorando, assim, o prognóstico materno e perinatal.

\section{ABSTRACT}

Objective: to assess the action of antiretroviral drugs on glycid metabolism and on the pancreas of pregnant Wistar rats. Methods: adult pregnant Wistar rats weighing 200-230g were used. Azidothymidine, lamivudine and nelfinavir were administered to the animals at doses 10 times higher than those administered to pregnant women. The animals were divided into seven groups of 10 animals, including a control group. The animals were sacrificed on the 21st day of pregnancy and glycemia, insulinemia, glucagonemia, free fatty acids (FFA) and hepatic glycogen were measured. Direct counts of the number of immunohistochemically labeled insulin- and glucagon-producing cells were used to determine pancreatic damage. Data were analyzed statistically by the Student's t-test comparing each treated group with the control group.

Results: increased serum glucagon (control group: $88.2 \mathrm{pg} /$ $\mathrm{ml}$; treated groups: 99.7-120.7 pg/ml) and reduced insulin (control group: $6.2 \mu \mathrm{IU} / \mathrm{ml}$; treated groups: $2.1-2.7 \mu \mathrm{IU} / \mathrm{ml}$ ) were observed in all groups treated with antiretroviral drugs after 21 days of pregnancy. There was no significant difference between the experimental groups and the control in glycemia, plasma FFA or hepatic glycogen. Also, there was no significant difference in number of insulin- and glucagon-producing cells between the treated groups and the control.

Conclusions: treatment of noninfected rats with antiretroviral drugs during pregnancy altered maternal glycid metabolism causing insulin decrease and glucagon elevation, with normal glycemia and unchanged number of pancreatic cells.

KEYWORDS: Antiretroviral drugs. Diabetes. HIV. AIDS.

\section{Referências}

1. Connor EM, Sperling RS, Gelber R, et al. Reduction of maternal-infant transmission of human immunodeficiency virus type 1 with zidovudine treatment. Pediatric AIDS Clinical Trial Group Protocol 076 Study Group. N Engl J Med 1994; 331:1173-80.

2. Tuomala RE, Shapiro D, Mofenson LM, et al. Antiretroviral therapy during pregnancy and the risk of an adverse outcome. N Engl J Med 2002; 346:1863-70.

3. Figueiró-Filho EA, Duarte G, Silva AAMR, et al. Efeito das drogas anti-retrovirais sobre as taxas de fertilidade de ratas Wistar. Rev Bras Ginecol Obstet 2002; 24:647-52.

4. European Collaborative Study. Exposure to antiretroviral therapy in utero or early life: the health of uninfected children born to HIV-infected women. J Acquir Immune Defic Syndr 2003; 32:380-7. 
5. Stein JH. Dyslipidemia in the era of HIV protease inhibitors. Prog Cardiovasc Dis 2003; 45:293-304.

6. Woerle HJ, Mariuz PR, Meyer C, et al. Mechanisms for the deterioration in glucose tolerance associated with HIV protease inhibitor regimens. Diabetes 2003; 52:918-25.

7. E1 Beitune P, Duarte G, Quintana SM, et al. Intolerância glicêmica e prognóstico perinatal em gestantes utilizando anti-retrovirais. Rev Bras Ginecol Obstet 2003; 25:465-71.

8. El Beitune P, Duarte G, Santos JE, Quintana SM, Figueiró-Filho EA, Marcolin AC. O uso de antiretrovirais em gestantes modifica o perfil lipídico? Rev Bras Ginecol Obstet 2003; 25:593-8.

9. Toltzis P, Marx CM, Kleinman N, Levine EM, Schmidt EV. Zidovudine-associated embryonic toxicity in mice. J Infect Dis 1991; 163:1212-8.

10.Rudge MVC, Calderon IMP, Ramos MD, Rodrigues MAM. Diabetes and experimental pregnancy in rats: course of maternal blood glucose levels and its repercussions on the blood glucose levels and pancreas of newborn pups. Braz J Med Biol Res 1995; 28:219-25.

11.Kanter M, Yoruk M, Koc A, Meral I, Karaca T. Effects of cadmium exposure on morphological aspects of pancreas, weights of fetus and placenta in streptozotocin-induced diabetic pregnant rats. Biol Trace Elem Res 2003; 93:189-200.

12.Damasceno DC, Volpato GT, Calderon IDP, Rudge MVC. Oxidative stress and diabetes in pregnant rats. Anim Reprod Sci 2002; 72:235-44.

13. Ghebremeskel K, Bitsanis D, Koukkou E, Lowy C, Poston L, Crawford MA. Liver triacylglycerols and free fatty acids in streptozotocin-induced diabetic rats have atypical $n-6$ and $n-3$ pattern. Comp Biochem Physiol C Toxicol Pharmacol 2002; 132:349-54.

14. Malaisse WJ, Ladriere L, Cancelas J, Acitores A, Villanueva-Penacarrillo ML, Valverde I. Pancreatic and hepatic glycogen content in normoglycemic and hyperglycemic rats. Mol Cell Biochem 2001; 219:45-9.

15. Chang KJ, Kwon W. Immunohistochemical localization of insulin in pancreatic beta-cells of taurine-supplemented or taurine-depleted diabetic rats. Adv Exp Med Biol 2000; 483:579-87.
16.Takatori A, Nishida E, Inenaga T, et al. Functional and histochemical analysis on pancreatic islets of APA hamsters with SZ-induced hyperglycemia and hyperlipidemia. Exp Anim 2002; 51:9-17.

17.Hsu SM, Raine L, Fanger H. Use of avidin-biotinperoxidase complex (ABC) in immunoperoxidase techniques : a comparison between $\mathrm{ABC}$ and unlabeled antibody (PAP) procedures. J Histochem Cytochem 1981; 29:577-80.

18.Ku SK, Lee HS, Lee JH. Immunohistochemical study of the pancreatic endocrine cells in the BALB/c mice: an unique distributional pattern of glucagon. J Vet Sci 2002; 3:167-73.

19.Dunbar JC, Brown A. Glucagon secretion by dispersed alpha cells enriched islets from streptozotocin treated hamsters in perifusion. Horm Metab Res 1984; 16:221-5.

20.Tanaka Y, Toyota N, Fujimoto K, et al. Immunohistochemical studies of fetal and maternal endocrine pancreases during pregnancy and the puerperium in streptozotocin-induced diabetic rats. Nippon Sanka Fujinka Gakkai Zasshi 1986; 38:1989-97.

21.Lucke S, Ziegler B, Weidlich K, Barowski U, Besch W, Hahn HJ. The effects of subdiabetogenic streptozotocin doses on rat beta cell volume and functions. Biomed Biochem Acta 1985; 44:167-71.

22.Bonner-Weir S, Trent DF, Honey RN, Weir GC. Responses of neonatal rat islets to streptozotocin: limited B-cell regeneration and hyperglycemia. Diabetes 1981 ; 30:64-9.

23.Guz Y, Nasir I, Teitelman G. Regeneration of pancreatic beta cells from intra-islet precursor cells in an experimental model of diabetes. Endocrinology $2001 ; 142: 4956-68$.

24.Marynissen G, Aerts L, Van Assche FA. The endocrine pancreas during pregnancy and lactation in the rat. J Dev Physiol 1983; 5:373-81.

25.Bonnevie-Nielsen V, Steffes MW, Lernmark A. A major loss in islet mass and B-cell function precedes hyperglycemia in mice given multiple low doses of streptozotocin. Diabetes 1981 ; 30:424-9.

26.Ziegler B, Lucke S, Besch W, Hahn HJ. Pregnancyassociated changes in the endocrine pancreas of normoglycaemic streptozotocin-treated Wistar rats. Diabetologia 1985; 28:172-5.

27.Garcia Carrapato MR. The offspring of gestational diabetes. J Perinat Med 2003; 31:5-11. 